\title{
Investigation of ternary subsystems of superalloys by thin-film combinatorial synthesis and high-throughput analysis
}

\author{
Dennis König ${ }^{1}$, Janine Pfetzing-Micklich ${ }^{2}$, Jan Frenzel ${ }^{2}$, and Alfred Ludwig ${ }^{1}$ \\ ${ }^{1}$ MEMS materials, Institute for Materials, Ruhr-Universität Bochum, 44801 Bochum, Germany \\ ${ }^{2}$ Materials Science and Engineering, Institute for Materials, Ruhr-Universität Bochum, 44801 Bochum, Germany
}

\begin{abstract}
A Co-Ti-W thin film materials library was fabricated by magnetron sputtering. By using automated high-throughput measurement techniques (resistance mapping, automated XRD measurements) and cluster analysis a yet unknown phase region was revealed. The existence region of the new ternary phase is close to the composition $\mathrm{Co}_{60} \mathrm{Ti}_{15} \mathrm{~W}_{25}$. In order to transfer the results from thin film to bulk material, a bulk sample was prepared by arc melting and subsequent heat treatment. Scanning electron microscopy and chemical microanalysis data support that a yet unknown ternary phase exists in the system Co-Ti-W.
\end{abstract}

\section{Introduction}

Ni-based superalloys are materials of choice for applications as turbine blades for airplane or stationary gas turbines due to their excellent high-temperature stability, creep behavior and chemical resistance [1-3]. In the course of the development of more efficient turbines, a temperature increase leads to an increase of the overall efficiency. This increase in temperature in turn leads to a demand for new materials which can withstand even higher temperatures than Ni-based superalloys are capable of. The change from Ni-based superalloys to Co-based superalloys could be an important step in this direction. For Co-based systems the desired $\gamma / \gamma^{\prime}$ microstructure was also observed [4]. However, the investigation of yet unknown alloy systems is very time consuming and cost intensive. Combinatorial materials science methods for the synthesis of materials libraries combined with highthroughput characterization techniques are efficient to study less-known or completely unknown material systems with the aim of identifying compositions with interesting properties or new phases [5-7]. In order to verify the adaptability of the thin film combinatorial methodology for superalloy subsystems, the $\mathrm{Cr}-\mathrm{Ni}$-Re system was studied at $1100^{\circ} \mathrm{C}$ by some of the present authors [8]. The system contains three solid solutions and a TCP intermetallic compound. The results found in the thin film materials library are in good agreement with results reported for bulk samples by other groups.

In this work we apply the thin film combinatorial materials science approach to study the Co-Ti-W system. This system is an important subsystem in Co-based superalloys [9-11] since both phases $\gamma$ and $\gamma$ ' are forming in the Co-Ti binary part. W plays an essential role in strength hardening, as well as stabilizing the $\mathrm{L}_{2}$ phase in the Co-Al-W subsystem [4]. So far, no experimental phase diagram of the Co-Ti-W system is available.

\section{Experimental methods}

Co-Ti-W materials libraries were fabricated on polycrystalline 4-inch diameter $\mathrm{Al}_{2} \mathrm{O}_{3}$ substrates by magnetron sputtering applying a wedge-type multilayer approach, with $120^{\circ}$ rotations between each layer, as described in [8, 12]. The substrate was heated to $300^{\circ} \mathrm{C}$ during deposition. Sputtering powers for $\mathrm{Co}$, $\mathrm{Ti}$, and $\mathrm{W}$ were set to $124 \mathrm{~W}$ DC, $159 \mathrm{~W}$ DC and $114 \mathrm{~W}$ DC, respectively. Ar was used as sputtering gas (purity $99.9999 \%, 2.7 * 10^{3}$ mbar, $40 \mathrm{sccm}$ ). Subsequent to sputter deposition the fabricated multilayer precursor structures were annealed at $950^{\circ} \mathrm{C}$ for $40 \mathrm{~h}$ in vacuum in order to initiate diffusion and phase formation, and finally transform the multilayer precursor structure into an alloy. In order to investigate the chemical composition spread existing on the thin film materials library, automated EDX measurements were performed. For these measurements a measurement grid with 342 measurement areas with regular distances of $4.5 \mathrm{~mm}$ in $\mathrm{x}$ - and $\mathrm{y}$-direction was used. The EDX analysis was performed using a JEOL 5800 LV scanning electron microscope (SEM) equipped with an Oxford Instruments INCA EDX-detector. Phase analysis was performed using X-ray diffraction (XRD) in Bragg-Brentano geometry. The measurements were carried out at room-temperature using a PANalytical X' Pert PRO MRD system (PiXcel detector, $\mathrm{Cu} \mathrm{K}_{\alpha}$ radiation, angular measurement range: $30^{\circ}-85^{\circ} 2 \Theta$, spot-size: $3 \mathrm{~mm} \times 5 \mathrm{~mm}$ ). Resistance mappings were performed using a custom-designed highthroughput test stand as described in [12]. For four-point electrical resistance measurements a measurement grid with 3084 measurement areas and regular distances of $1.5 \mathrm{~mm}$ between the measurement areas was used. For statistics, each measurement area was measured three times. These values were averaged and were used for further analysis. The measurements were performed at 


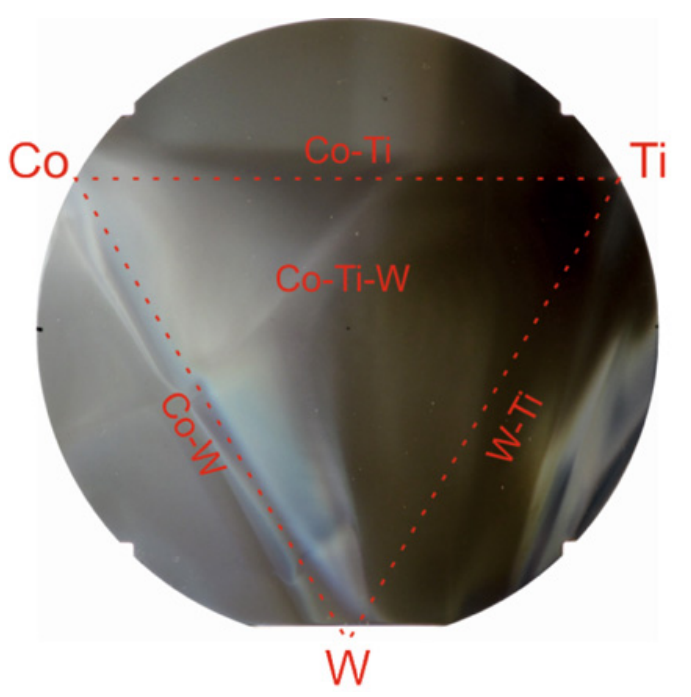

Figure 1. Photograph of a thin film Co-Ti-W materials library deposited on a 4 inch alumina substrate after annealing in vacuum at $950{ }^{\circ} \mathrm{C}$ for $40 \mathrm{~h}$. The binary and ternary composition regions are indicated, as well as the position of the sputter sources.

room-temperature applying a measurement current of $100 \mathrm{~mA}$.

In order to verify results obtained through thin film combinatorial materials science, also bulk samples were prepared. Co-Ti-W ingots of different compositions and a weight of $20 \mathrm{~g}$ were prepared by arc melting and subsequent homogenization annealing at $1100^{\circ} \mathrm{C}$ for $24 \mathrm{~h}$ in evacuated quartz capsules. The corresponding microstructures were characterized through SEM and EDX. All details on bulk sample preparation and SEM characterization are given in $[13,14]$.

\section{Results and discussion}

Figure 1 depicts a photograph of the Co-Ti-W materials library after heat treatment. After the heat treatment lines and striations are visible on the thin film materials library, which are first indications for phase formation and different phase regions.

However, this optical information is not sufficient. Fig. 2 shows the range of compositions being present on the materials library (determined by automated EDX measurements). The materials library covers about $40 \%$ of the ternary system: Ti contents from 0 at. $\%-40$ at. $\%$ are covered, whereas $\mathrm{W}$ contents from 0 at.\% -52 at. $\%$ and Co contents from 61 at.\% to 95 at.\% are covered. In the fabricated compositional region the interesting composition of $\mathrm{Co}_{3} \mathrm{Ti}$ is included. In order to identify possible locations of phase boundaries and phase areas high-throughput resistance measurements were performed.

The working hypothesis is that single phase regions could show a lower resistivity compared to mixed phase regions. Figure 3 depicts the results gained from the highthroughput resistance mapping. The resistance values are visualized color-coded as a function of the position on the wafer.

It is obvious that close to the center of the materials library there is an extended region showing relatively

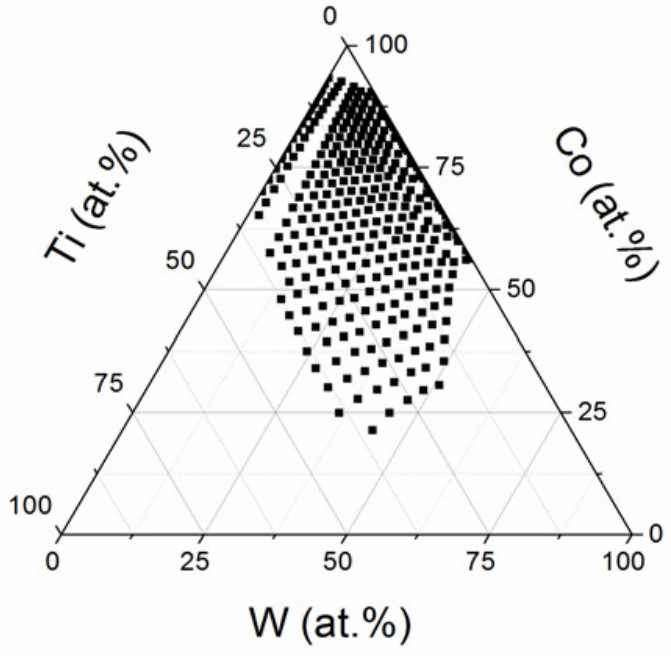

Figure 2. Ternary composition diagram, indicating the compositional region covered by the Co-Ti-W materials library with black squares (EDX measurement areas).

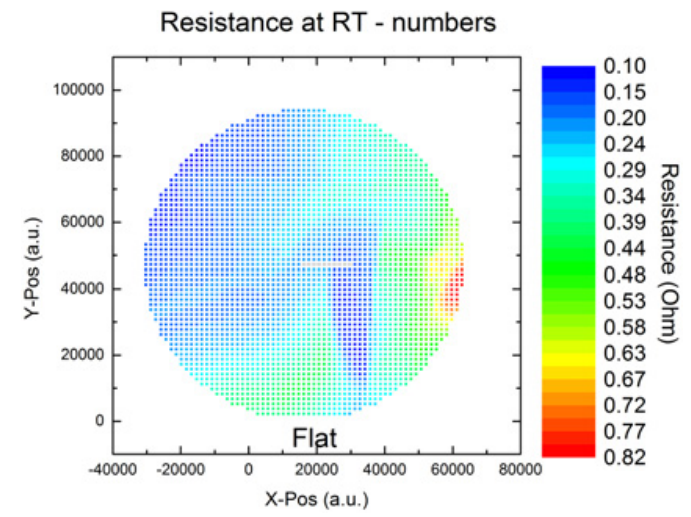

Figure 3. Visualization of the resistance mapping of the Co-Ti$\mathrm{W}$ materials library. The dark blue area with low resistance values close to the middle of the materials library indicates the region of a potentially new ternary phase.

low resistance values of only $\sim 0.1 \Omega$. This region of low resistance coincides well with a dark shaded region visible in Fig. 1. In order to check if this spatially confined low-resistance region is correlated with a new phase, the resistance data presented in Fig. 3 are plotted in a ternary composition diagram in Fig. 4. Here, the low-resistance region from Fig. 3 also shows up as a coherent region of low resistance with sharp boundaries to the adjacent regions of higher resistance. This clear and confined change of the resistance values is an indication for a phase boundary.

However, resistance measurements can only indicate a possible composition region in which a new phase exists. In order to prove the existence of a new phase and the distribution of known and unknown phases on the materials library, automated XRD measurements on all 342 measurement regions were performed. The data gained from these measurements was analyzed by a special software (XRD suite) which is suitable for the analysis of hundreds of diffractograms [15]. The diffractograms are grouped by similarity cluster analysis thus giving 


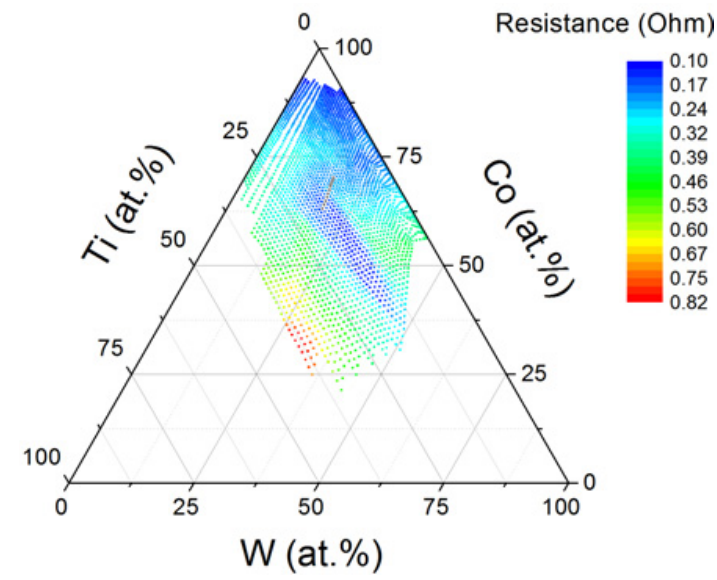

Figure 4. Resistance mapping data plotted color-coded in the ternary composition diagram.

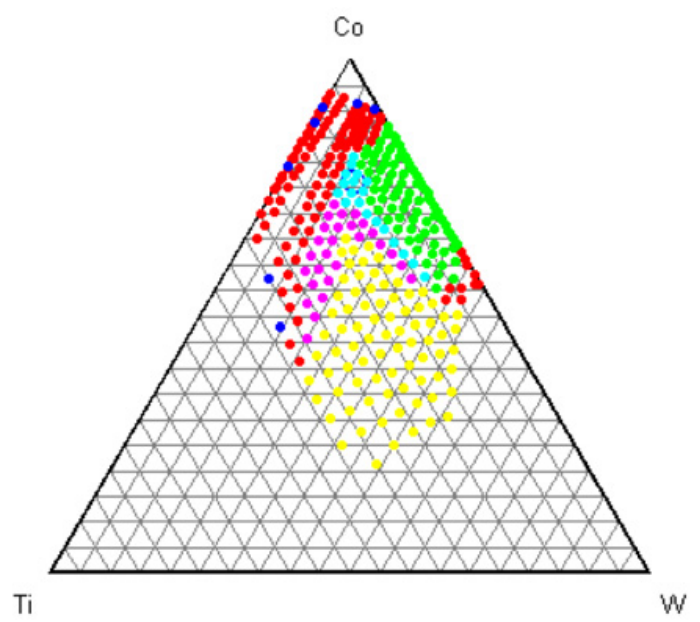

Figure 5. Results of the cluster analysis of all XRD diffractograms of the Co-Ti-W materials library. The light blue area coincides well with the low-resistance area revealed using the resistance mapping.

indications for different phase regions. Figure 5 depicts the results of the cluster analysis. Six clustered regions are visible. The region marked with light blue coincides well with the region visible from the resistance data present in the ternary composition diagram.

Using all data from the automated XRD measurements a diffraction intensity distribution as a function of chemical composition plot was derived using XRD suite software (Fig. 6): in a) the composition-dependent intensity distribution for a fixed diffraction angle of $43.5^{\circ} 2 \Theta$ is depicted.

This angle was chosen since it only appears for chemical compositions located in the low resistance region in Fig. 4 and thus in the proposed existence range of the eventually new phase. As visible in Fig. 6 there is only a significant intensity for this diffraction angle in the region marked light blue in Fig. 5.

By combining all these indications from resistance and automated XRD mappings it is concluded that a yet unknown phase is present for chemical compositions around $\mathrm{Co}_{60} \mathrm{Ti}_{15} \mathrm{~W}_{25}$ located in the light blue area in Fig. 5.

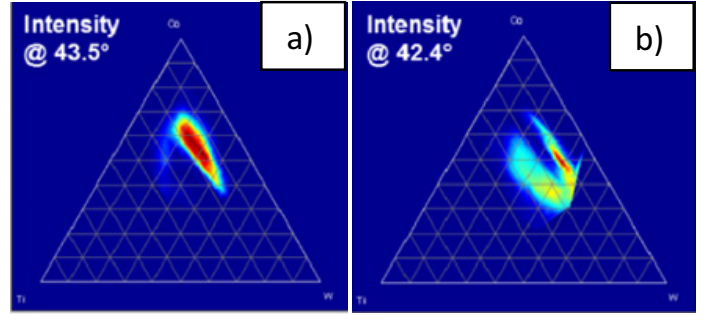

Figure 6. Diffraction intensity plots for fixed diffraction angles. (a) $43.5^{\circ}$, belonging to the potentially new ternary phase, (b) $42.4^{\circ}$, clearly indicating the presence of a phase region boundary.

In order to support this assumption a diffraction intensity distribution for another fixed Bragg angle $\left(42.4^{\circ} 2 \Theta\right)$ was determined. This is presented in the right part of Fig. 6. For this angle it becomes clear that there is no diffraction intensity in that region where diffraction for an angle of $43.5^{\circ} 2 \Theta$ only occurs. This strengthens the proposition that the proposed region is in fact a yet unknown phase region.

Figure 7 shows SEM micrographs of a $\mathrm{Co}_{68} \mathrm{Ti}_{22} \mathrm{~W}_{10}$ bulk sample. This composition lies within the region of interest that was identified (Fig. 6a). Figure 7a shows an overview of the microstructure, and a micrograph with higher magnification is given in Fig. 7b. The different regions marked 1, 2 and 3 were characterized by EDX, and the corresponding chemical compositions are given in Table 1. The backscatter contrast allows distinguishing three different phases. Figure 7a shows that there is a dendritic phase (1) with a bright contrast, which mainly consists of $\mathrm{W}$. This phase is surrounded by a darker phase (2) with significantly lower W concentration. This phase again is surrounded by a dark region with lamellar character and low $\mathrm{W}$ concentration. It can be concluded that the W-rich phase (1) has formed in the early stages of the solidification while the other phases formed at lower temperatures. Nevertheless, it is difficult to reconstruct all relevant microstructural evolution processes since the structures shown in Fig. 7 have a quite complex character.

Furthermore, it is likely that the material is still far away from equilibrium, and that a longer homogenization annealing would result in a more homogeneous distribution of $\mathrm{W}$ as well as in changes of phase fractions. Therefore, further work is required to characterize microstructures of bulk Co-Ti-W alloys.

However, the results of the present study clearly show that a ternary phase (region 2 in Fig. 7a) exists in the system Co-Ti-W, which has a composition matching previous results obtained by thin film combinatorial materials science methods (see Figs. 4-6).

\section{Conclusion and outlook}

A Co-Ti-W thin film materials library was fabricated using combinatorial magnetron sputtering. After annealing, striations and lines appear on the materials library indicated phase formation. Room-temperature resistance measurements reveal a clearly separated area of low 
Table 1. Chemical compositions (at.\%) of regions 1, 2 and 3 highlighted in Fig. 7.

\begin{tabular}{|l|l|l|l|}
\hline Phase/ region & Co & Ti & W \\
\hline $\mathbf{1}$ & 3.2 & 2.5 & 94.3 \\
\hline $\mathbf{2}$ & 57.3 & 12.7 & 30.0 \\
\hline $\mathbf{3}$ & 68.6 & 25 & 6.4 \\
\hline
\end{tabular}
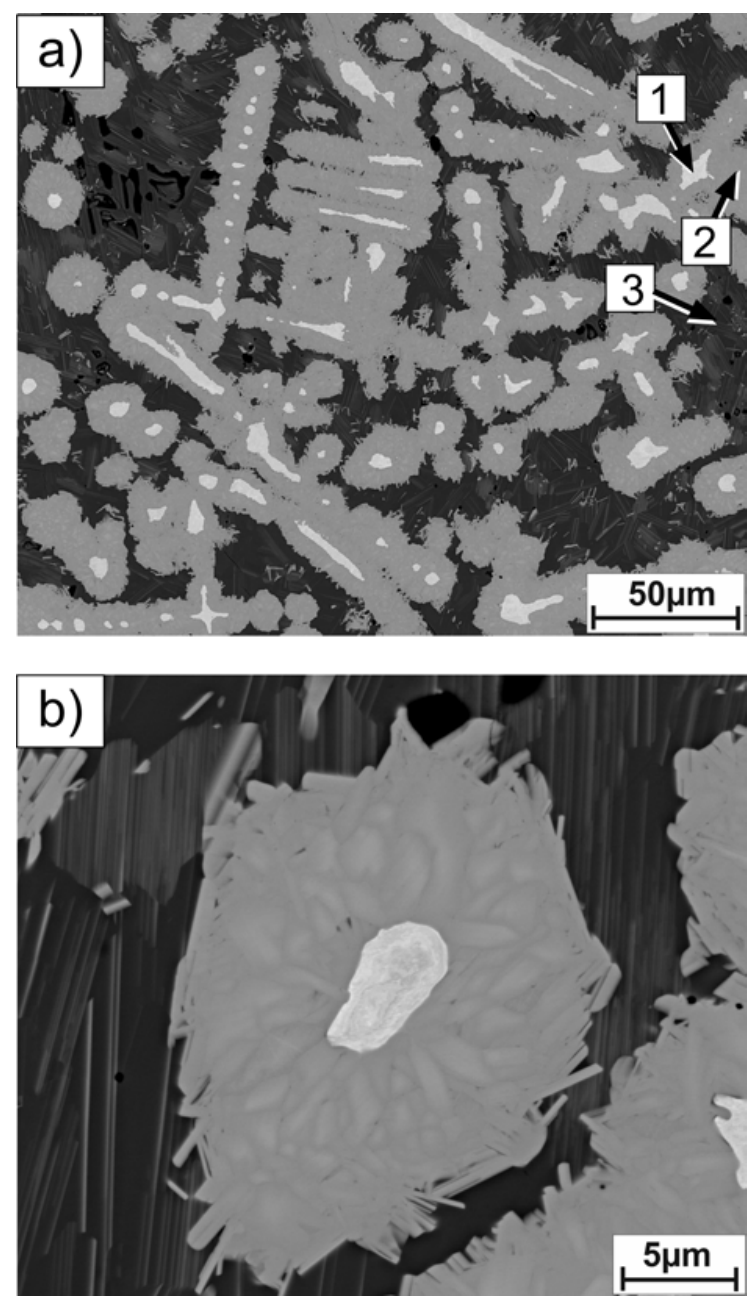

Figure 7. SEM back scatter micrographs of a bulk $\mathrm{Co}_{68} \mathrm{Ti}_{22} \mathrm{~W}_{10}$ sample; a) overview image with different regions that were analyzed by EDX (see Table 1); b) microstructure at higher magnification.

resistance within the materials library. This area shows indications to be a compositional region containing a new ternary phase. This was verified with high-throughput
XRD measurements, which were analyzed by peak intensity mapping and cluster analysis. Finally, the results from the thin film materials library were up-scaled to bulk. The results of the analysis of the bulk sample support the assumption of a new ternary phase in the Co-Ti-W system.

This work was supported by the German Research Foundation (DFG) through project B5 in the framework of SFB / Transregio 103. Dr. A. Janghorban is acknowledged for his initial contributions to this work.

\section{References}

[1] Z. Huda, P. Edi, Materials \& Design, 46, 552, (2013)

[2] C.T. Sims, N.S. Stoloff, W.C. Hagel (Eds.), Superalloys II: High Temperature Materials for Aerospace and Industrial Power, second ed., (Wiley Blackwell, New York, 1987)

[3] R.C. Reed, The Superalloys: Fundamentals and Applications, (Cambridge University Press, 2006)

[4] J. Sato, T. Omori, K. Oikawa, I. Ohnuma, R. Kainuma, K. Ishida, Science, 312, 90-91, (2006)

[5] D. König, R. Zarnetta, A. Savan, H. Brunken, A. Ludwig, Acta Mater., 59, 3267-3275, (2011)

[6] P. J. S. Buenconsejo, R. Zarnetta, D. König, A. Savan, S. Thienhaus, A. Ludwig, Adv. Funct. Mater., 21, 113-118, (2011)

[7] S. Hamann, M. E. Gruner, S. Irsen, J. Buschbeck, C. Bechtold, I. Kock, S. G. Mayr, A. Savan, S. Thienhaus, E. Quandt, S. Fähler, P. Entel, A. Ludwig, Acta Mater., 58, 5949-5961, (2010)

[8] A. Janghorban, J. Pfetzing-Micklich, J. Frenzel, A. Ludwig, Adv. Eng. Mater., doi: 10.1002/adem. 201300430

[9] A. Bauer, S. Neumeier, F. Pyczak, R. F. Singer, M. Göken, Mater. Sci. Eng. A, 550, 333-341, (2012)

[10] A. Bauer, S. Neumeier, F. Pyczak, M. Göken, Scripta Mater., 63, 1197-1200, (2010)

[11] A. Suzuki, T. Pollock, Acta Mater., 56, 1288-1297, (2008)

[12] A. Ludwig, R. Zarnetta, S. Hamann, A. Savan, S. Thienhaus, Inter. J. Mater. Res., 99, 1144, (2008)

[13] J. Frenzel, E. P. George, A. Dlouhy, Ch. Somsen, M.F.-X. Wagner, G. Eggeler, Acta Mater. 58, 3444 3458, (2010)

[14] M. Rahim, J. Frenzel, M. Frotscher, J. PfetzingMicklich, R. Steegmüller, M. Wohlschlögel, H. Mughrabi, G. Eggeler, Acta Mater. 61, 10, 3667, (2013)

[15] C. J. Long et al., Rev. Sci. Instrum.,78, 072217, (2007) 